\title{
tic\&société
}

Vol. 14, $\mathbf{N}^{\circ}$ 1-2 | 1er semestre 2020 - 2ème semestre 2020

Mutations numériques de la musique : des

contradictions à analyser

\section{Les téléphones mobiles, un outil de désinformation? La circulation des informations peu fiables dans Twitter lors de la campagne présidentielle française de 2017}

Mobile phones as disinformation tools? Unreliable Information on Twitter

during the 2017 French presidential campaign

Teléfonos móviles, ¿una herramienta de desinformación? La circulación de

informaciones poco fiables en Twitter durante la campaña presidencial francesa

de 2017

Julien FIGEAC, Pierre RATINAUD, Nikos SMYRNAIOS, Guillaume

CABANAC, Ophélie FRAISIER-VANIER, Tristan SALORD et Fanny

SEFFUSATTI

\section{OpenEdition}

Édition électronique

URL : http://journals.openedition.org/ticetsociete/5413

DOI : 10.4000/ticetsociete. 5413

Éditeur

Association ARTIC

Édition imprimée

Pagination : 375-403

Référence électronique

Julien FIGEAC, Pierre RATINAUD, Nikos SMYRNAIOS, Guillaume CABANAC, Ophélie FRAISIER-VANIER,

Tristan SALORD et Fanny SEFFUSATTI, « Les téléphones mobiles, un outil de désinformation? La circulation des informations peu fiables dans Twitter lors de la campagne présidentielle française de 2017 », tic\&société [En ligne], Vol. 14, № 1-2 | 1er semestre 2020 - 2ème semestre 2020, mis en ligne le 11 novembre 2020, consulté le 23 février 2021. URL : http://journals.openedition.org/ticetsociete/ 5413 ; DOI : https://doi.org/10.4000/ticetsociete.5413 
tic\&société - 14(1-2), 2020

\title{
Les téléphones mobiles, un outil de désinformation? La circulation des informations peu fiables dans Twitter lors de la campagne présidentielle française de 2017
}

\begin{abstract}
Julien Figeac
Julien Figeac est sociologue, chargé de recherche au CNRS au LISST (UMR 5193) à l'Université de Toulouse. Ses travaux interdisciplinaires traitent des usages des technologies de l'information et de la communication. II a publié plusieurs articles sur les pratiques d'information, les usages des médias sociaux, les sociabilités et les réseaux de relations en ligne, les formes connectées de participation politique. julien.figeac@univ-tlse2.fr
\end{abstract}

\section{Pierre Ratinaud}

Pierre Ratinaud est maître de conférences en sciences de l'éducation à l'Université de Toulouse, membre du LERASS. Ses travaux portent sur la dynamique des systèmes de représentations sociales et professionnelles et sur l'analyse statistique des données textuelles. II est également le développeur du logiciel libre IRaMuteQ, pour l'analyse multidimensionnelle des textes et des questionnaires. ratinaud@univ-tlse2.fr

\section{Nikos Smyrnaios}

Nikos Smyrnaios est maître de conférences en sciences de l'information et de la communication à l'Université de Toulouse, membre du LERASS. Ses sujets de recherche concernent l'économie politique des médias en ligne, le journalisme numérique et les usages politiques des réseaux sociaux numériques. Son ouvrage Les Gafam contre l'internet - Une économie politique du numérique a été publié en France en 2017 et traduit en anglais en 2018. nicolaos.smyrnaios@iut-tlse3.fr 
Les téléphones mobiles, un outil de désinformation ? La circulation des informations peu fiables dans Twitter lors de la campagne présidentielle française de 2017

\section{Guillaume Cabanac}

Guillaume Cabanac est maître de conférences habilité à diriger des recherches (HDR) en informatique à l'Université de Toulouse, membre de l'Institut de recherche en informatique de Toulouse (IRIT UMR 5505 CNRS). Ses travaux interdisciplinaires se positionnent à la croisée de l'analyse des réseaux sociaux numériques et de la scientométrie - désignant l'étude quantitative de la science et de l'innovation. II siège au Comité national du CNRS en qualité de membre nommé du Conseil scientifique de l'Institut des sciences humaines et sociales (InSHS). guillaume.cabanac@univ-tlse3.fr

\section{Ophélie Fraisier-Vannier}

Ophélie Fraisier-Vannier est docteure en informatique, postdoctorante à I'IRIT à I'Université de Toulouse. ophelie.fraisier-vannier@univ-tlse3.fr

\section{Tristan Salord}

Tristan Salord est sociologue, ingénieur d'études à I'IRIT à l'Université de Toulouse. Ses terrains pluridisciplinaires le positionnent dans le champ des digital humanities, où il mobilise des méthodes issues à la fois des approches qualitatives propres aux sciences humaines et des approches Big Data. $\underline{\text { tristan.salord@irit.fr }}$

\section{Fanny Seffusatti}

Fanny Seffusatti est sociologue, assistante de recherche au CNRS au LISST (UMR 5193) à I'Université de Toulouse. sef.fanny@gmail.com 
Julien FIGEAC, Pierre RATINAUD, Nikos SMYRNAIOS, Guillaume CABANAC, Ophélie FRAISIER-VANNIER, Tristan SALORD et Fanny SEFFUSATTI

\title{
Les téléphones mobiles, un outil de désinformation? La circulation des informations peu fiables dans Twitter lors de la campagne présidentielle française de 2017
}

\begin{abstract}
Résumé : Cette recherche analyse les modes de propagation des sources d'information peu fiables au sein de Twitter lors de l'élection présidentielle française de 2017 en évaluant plus précisément le rôle des téléphones mobiles dans les échanges d'idées et de contenus. Elle montre que l'usage de l'application mobile de Twitter défavorise le partage d'articles publiés par les organes de presse au profit des informations produites par des sources alternatives, notamment des blogues ou des sites Web de militants. Comme ces sources alternatives relaient davantage de désinformation, les téléphones mobiles tendent à favoriser le partage d'informations peu fiables. Cependant, cet effet des téléphones mobiles dans le phénomène de la désinformation vient avant tout amplifier les pratiques des communautés politiques, notamment le Front national, qui organisent par ailleurs leurs débats autour d'un nombre plus important de références peu fiables.
\end{abstract}

Mots-clés: réseaux socionumériques, téléphone mobile, presse, désinformation, participation politique.

\section{Mobile phones as disinformation tools? Unreliable Information on Twitter during the 2017 French presidential campaign}

\begin{abstract}
This research analyses the spread of unreliable information on Twitter during the 2017 French presidential campaign, focusing on the effects of mobile phones of
\end{abstract}


Les téléphones mobiles, un outil de désinformation ? La circulation des informations peu fiables dans Twitter lors de la campagne présidentielle française de 2017

participation in the exchange of ideas and content. It shows that the Twitter application discriminates against the sharing of articles published by the mainstream press in favor of information produced by alternative sources, in particular blogs or activists' websites. As these sources relay more disinformation, users of mobile phones appear to promote the spread of unreliable information. The effects of this information-sharing behavior are particularly salient within political communities that also organize their debates around a larger number of unreliable sources, such as the far right.

Keywords: social media, mobile phone, press, misinformation, political participation.

\title{
Teléfonos móviles, ¿una herramienta de desinformación? La circulación de informaciones poco fiables en Twitter durante la campaña presidencial francesa de 2017
}

\begin{abstract}
Resumen: Esta investigación analiza los modos de propagación de fuentes de información poco fiables dentro de Twitter durante la elección presidencial francesa de 2017. En concreto, se evalúa el papel de los teléfonos móviles en el intercambio de ideas y de contenidos. Muestra que el uso de la aplicación móvil de Twitter perjudica el intercambio de artículos publicados por los medios de comunicación clásicos en beneficio de la información producida por fuentes alternativas, en particular blogs o sitios web de activistas. En la medida en que estas fuentes alternativas transmiten más desinformación, los teléfonos móviles tienden a favorecer el intercambio de información poco fiable. Sin embargo, este efecto de los teléfonos móviles en el fenómeno de la desinformación amplifica las prácticas de las comunidades políticas, en particular del Frente Nacional, que también organizan sus debates en torno a un número, cada vez mayor, de referencias poco fiables.
\end{abstract}

Palabras claves: redes socio-digitales, teléfono móvil, prensa, desinformación, participación política. 


\section{Julien FIGEAC, Pierre RATINAUD, Nikos SMYRNAIOS, Guillaume CABANAC, Ophélie FRAISIER-VANNIER, Tristan SALORD et Fanny SEFFUSATTI}

La fabrication des fausses informations n'a pas débuté avec Internet et Twitter. Si l'on remonte le fil du temps de l'histoire, on s'aperçoit rapidement à quel point la problématique de la désinformation, qui a été fortement médiatisée par l'anglicisme fake news, est apparue très tôt (Uberti, 2016). Cette histoire nous rappelle que les fausses informations ne sont pas forcément l'affaire d'individus cyniques, radicalisés et malveillants. Elles renvoient aussi aux nombreuses bourdes commises par des journalistes de plus en plus incités à s'inscrire dans la recherche de scoops.

On ne peut pas comprendre l'ampleur de la problématique des fake news, et mesurer leurs effets sur nos sociétés, si on réduit le sens de cet anglicisme polysémique à son acceptation la plus radicale, celle de la "désinformation ». Wardle et Derakhshan (2017) définissent cette notion comme une fausse information, produite délibérément pour porter préjudice à une personne, un groupe social, une organisation ou un pays. Ils la distinguent de la « mésinformation », comprise comme une fausse information produite cette fois sans intention de causer du tort, que ce soit par un professionnel de l'information ou par un citoyen internaute. La prise en compte de cette "simple " mésinformation est essentielle, car elle incite à prendre continuellement la mesure du caractère stratégique ou non d'un usage d'Internet dans la propagation de informations erronées.

II est important de déconstruire ainsi l'anglicisme fake news tant son usage est galvaudé, notamment au sein des articles scientifiques (Tandoc, Lim et Ling, 2018). En effet, les premiers travaux ont utilisé cette notion pour analyser des phénomènes précis de désinformation au sein de Twitter, comme la propagation de rumeurs scientifiques liées à la découverte du Boson de Higgs (De Domenico et al., 2013) ou à un tremblement de terre à Haïti (Oh, Kwon et Rao 2010). Une autre perspective de recherche a consisté à analyser comment les résultats des requêtes de Google ont pu favoriser, par rapport à Bing, le moteur de recherche concurrent développé par Microsoft, une certaine mésinformation autour du virus Zika (Venkatraman et al., 2016). 
Les téléphones mobiles, un outil de désinformation ? La circulation des informations peu fiables dans Twitter lors de la campagne présidentielle française de 2017

Ces différentes perspectives d'analyse de la désinformation ont été davantage diversifiées suite à la campagne présidentielle états-unienne de 2016 lorsque des recherches ont évalué le rôle des fausses informations dans l'accession au pouvoir du candidat Trump. À la différence des travaux pionniers, ces études ne se sont pas focalisées sur la propagation d'une rumeur en particulier, mais sur un ensemble de rumeurs en exploitant les milliers de fausses informations recensées par les sites de fact-checking. Cette perspective de recherche, davantage quantitative, a ainsi été suivie par des chercheurs pour évaluer dans quelle mesure de fausses informations ont pu favoriser la candidature de Trump, notamment auprès de ses partisans (Allcott et Gentzkow, 2017). Elle a plus généralement révélé que la vitesse de diffusion des informations frauduleuses ou erronées est plus rapide au sein de Twitter que celle des informations vérifiées (Vosoughi, Roy et Aral, 2018).

Quelques mois plus tard, les regards se sont tournés vers la campagne présidentielle française de 2017 et la manière dont les producteurs de fausses informations pouvaient chercher à influencer les débats, comme cela a par exemple été le cas lorsque le candidat Emmanuel Macron a été accusé de détenir un compte bancaire situé à l'étranger ${ }^{1}$. Des observateurs du champ journalistique ont identifié de nombreux exemples de désinformation au sein des médias sociaux avant d'insister sur le rôle des sources d'informations " non traditionnelles » et alternatives ${ }^{2}$, notamment les sites Web de militants politiques, dans la mesure où elles ont joué un rôle central dans la propagation des fausses informations. Dans le cadre de cet article $^{3}$, nous voudrions prolonger leurs observations en analysant les usages et la circulation de ces sources d'information alternatives dans Twitter pour évaluer dans quelle mesure elles ont pu compléter, voire concurrencer les contenus publiés par les organes de presse.

II s'agit de prolonger les travaux antérieurs qui ont exploré la

\footnotetext{
1 CrossCheck (2017, 5 mai). Did Emmanuel Macron Open an Offshore Account?. CrossCheck. Repéré à https://crosscheck.firstdraftnews.org/checkedfrench/emmanuel-macron-open-offshore-account/

2 Cf. Haski, P. (2017). Patterns of disinformation in the 2017 French Presidential Election, Report. Repéré à https://www.bakamosocial.com/frenchelection/

${ }^{3}$ Ce travail a été réalisé dans le cadre du projet ANR LisTIC portant la référence ANR16-CE26-0014-01.
} 


\section{Julien FIGEAC, Pierre RATINAUD, Nikos SMYRNAIOS, Guillaume CABANAC, Ophélie FRAISIER-VANNIER, Tristan SALORD et Fanny SEFFUSATTI}

propagation des fausses informations au sein des espaces numériques francophones, que ce soit dans les blogues personnels (Venturini et al., 2018) ou sur Twitter (Gaumont, Panahi et Chavalarias, 2018). Nous allons cibler, ici, les usages de Twitter en mettant plus précisément l'accent sur le rôle des outils utilisés pour consulter ce média, notamment les téléphones mobiles, dans la propagation de la désinformation : l'utilisation de l'application mobile de Twitter peut-elle favoriser la propagation des fausses informations ? Le fait de naviguer dans Twitter depuis un navigateur Web installé sur un ordinateur limite-t-il au contraire les risques de relayer de la désinformation? Nous proposons dès lors d'évaluer si les utilisateurs qui ont le plus partagé de la désinformation durant l'élection présidentielle française de 2017 ont davantage utilisé l'application mobile de Twitter ou s'ils ont plutôt navigué dans la plateforme de ce média social via un ordinateur.

Dans la partie suivante, nous allons référencer des travaux sur la désinformation pour positionner l'intérêt et la singularité de cet article. Suite à cela, la présentation des résultats se fera en deux temps. Tout d'abord, nous mesurerons la part des diverses sources d'information (presse journalistique, sites politiques, pages personnelles, etc.) partagées par les principales communautés politiques françaises (LFI, PS, REM, LR et FN ${ }^{4}$ ) depuis les plateformes fixes et mobiles de Twitter. Puis, nous évaluerons plus précisément quelle est la part des sources d'information "fiables " et des sources "peu fiables " au sein des différents contenus partagés par les différentes communautés politiques. L'objectif consistera à mesurer si le nombre de sources d'information fiables et peu fiables varie en fonction des communautés politiques et s'il est différent en fonction des plateformes utilisées, que ce soit l'application mobile ou la plateforme Internet de Twitter.

\footnotetext{
${ }^{4}$ Nous parlerons ici du Front national puisque ce parti a été rebaptisé Rassemblement national $(R N)$ le $1^{\text {er }}$ juin 2018, après la réalisation de cette recherche.
} 
Les téléphones mobiles, un outil de désinformation ? La circulation des informations peu fiables dans Twitter lors de la campagne présidentielle française de 2017

\section{La téléphonie mobile dans la propagation des sources d'information peu fiables}

Les recherches sur la propagation de la désinformation au sein des médias sociaux évacuent systématiquement le rôle du support technologique (téléphone mobile ou ordinateur) ou du service (application mobile ou plateforme Internet). Pourtant, la sociologie des usages a déjà mis en avant l'importance de ces intermédiaires et du design de leurs interfaces dans la structuration des pratiques d'information (Figeac, 2007) et dans les usages des réseaux socionumériques (Figeac et Chaulet, 2018 ; Tufekci, 2019). Des études expérimentales ont même récemment démontré que la petite taille des écrans de téléphones diminuait le degré de vigilance des internautes en les incitant à ouvrir plus facilement des contenus malicieux (Vishwanath, 2016). II est dès lors central d'essayer d'évaluer comment les terminaux (fixes ou mobiles) utilisés pour consulter les sites Web peuvent influencer l'audience et la réception des fausses informations (Nelson et Taneja, 2018). Cette question est d'autant plus centrale depuis que les utilisateurs consultent essentiellement les médias sociaux depuis leurs applications mobiles (Smith et Page, 2015). II est pertinent de chercher à saisir si les logiques d'usage nomades de ces applications peuvent infléchir les logiques de participation à ces réseaux socionumériques, et plus précisément si ces usages mobiles peuvent favoriser la propension des utilisateurs à (re)tweeter, volontairement ou non, de la désinformation.

Cette question est également importante, car les téléphones mobiles occupent une place de plus en plus essentielle dans les pratiques d'information des individus (Westlund, 2015). Ce phénomène est d'ailleurs plus courant dans les pays, de l'Afrique subsaharienne par exemple, où les individus ont uniquement accès à Internet et aux informations politiques de la presse en ligne par l'intermédiaire de leurs téléphones mobiles (Aker, Collier et Vicente, 2017). Si les téléphones mobiles jouent un rôle de plus en plus central dans la manière dont les individus consultent des informations, et notamment des informations politiques, il va de soi qu'ils jouent de fait un rôle de plus en plus important dans la propagation de la désinformation. II s'agit du moins de la question de recherche à laquelle cet article va tenter de répondre: en comparaison avec la plateforme Web de Twitter, quelle place l'application mobile de Twitter a-t-elle 


\section{Julien FIGEAC, Pierre RATINAUD, Nikos SMYRNAIOS, Guillaume CABANAC, Ophélie FRAISIER-VANNIER, Tristan SALORD et Fanny SEFFUSATTI}

occupée dans la diffusion de la désinformation lors de la campagne présidentielle française de 2017 ?

Cet article s'efforcera également de répondre à une seconde question pertinente à poser au regard de l'état des connaissances de la littérature scientifique. Plusieurs chercheurs rejoignent, non sans prudence, le constat dressé par des chercheurs de Facebook selon lequel la désinformation reste un phénomène marginal puisqu'elle représente moins de $1 \%$ des contenus "civiques " publiés au sein de ce média social (Weedon, Nuland et Stamos, 2017). Contrairement à une idée reçue, les fausses informations rencontrent bien une très faible audience (Nelson et Taneja, 2018). Ce qui compte avant tout, c'est la réception que les usagers font de ces fausses nouvelles, la manière dont ils les décodent et dont elles entrent en résonance avec leurs opinions politiques. Ils peuvent adhérer au message d'une fausse information, ils peuvent aussi le négocier et se le réapproprier, ou ils peuvent au contraire le rejeter, voire chercher à s'y opposer (Wardle et Derakhshan, 2017). Leur attitude varie énormément selon leur profil sociodémographique et leur conviction politique. Des travaux montrent ainsi que la circulation des fausses informations au sein de Twitter (Grinberg et al., 2019) ou de Facebook (Guess, Nagler et Tucker, 2019) est majoritairement le fait d'individus fortement politisés ; il s'agit plutôt de sympathisants républicains âgés de plus de 65 ans.

Cette dimension du positionnement des individus et des communautés politiques sur l'échiquier politique est centrale pour comprendre la propagation de la désinformation dans les médias sociaux. En France, il a effectivement été montré que la circulation des fausses informations est plus importante au sein des communautés politiques de la droite (Les Républicains) et de l'extrême droite (FN) (Gaumont, Panahi et Chavalarias, 2018). Dans le cadre de cet article, nous cherchons à prolonger ce résultat. Après avoir évalué si la propagation de la désinformation est plus fortement associée à l'usage de l'application mobile de Twitter, il s'agira d'observer les éventuelles différences entre les principales communautés politiques françaises: peut-on considérer que les formes de participation développées à partir de l'application mobile de Twitter tendent à amplifier la tendance de certaines communautés politiques, en l'occurrence Les Républicains et le 
Les téléphones mobiles, un outil de désinformation ? La circulation des informations peu fiables dans Twitter lors de la campagne présidentielle française de 2017

Front national, à faire circuler un nombre plus important de fausses informations?

\section{Méthode}

\subsection{Collecte et échantillonnage des données}

Afin d'étudier la circulation des fausses informations dans Twitter pendant la présidentielle de 2017, nous avons collecté un large corpus de tweets ${ }^{5}$ contenant des mots-clés relatifs aux cinq principaux candidats à l'élection. II s'agit des candidats qui ont réuni plus de $90 \%$ des suffrages au premier tour: Jean-Luc Mélenchon (LFI), Benoît Hamon (PS), Emmanuel Macron (LREM), François Fillon (LR) et Marine Le Pen (FN). Cette collecte des tweets a été réalisée entre le 25 novembre 2016 et le 12 mai 2017, soit cinq jours après le second tour de l'élection.

L'échantillon ainsi collecté se compose de 38346765 tweets et de retweets émis par 2163812 comptes différents. Ce jeu de données a été normalisé, vérifié manuellement puis mis en accès libre (Fraisier et al., 2018). Une fois en possession de cet échantillon de (re)tweets, il a été nécessaire de détecter les communautés politiques dans le but de pouvoir analyser la circulation des sources d'information au sein de chacune d'elle. Pour atteindre cet objectif, nous avons tout d'abord utilisé une méthode de détection automatique de communautés, puis nous avons procédé à une identification manuelle et qualitative de ces communautés afin de les nommer plus précisément.

La méthode de détection automatique a dans un premier temps permis d'identifier les différentes communautés politiques de notre corpus de tweets à l'aide de l'algorithme de Louvain. Cette procédure a permis d'agréger des groupes de comptes afin de nous permettre, dans un second temps, d'identifier et de nommer qualitativement les différentes communautés politiques émergeant du réseau de tweets (Smyrnaios et Ratinaud, 2014). Nous avons nommé ces communautés en identifiant notamment les comptes Twitter qui occupent une place centrale dans le réseau. Par exemple, nous avons isolé la communauté politique

${ }^{5}$ La collecte de données a été effectuée à l'aide de la plateforme OSIRIM qui est administrée par l'Institut de recherche en informatique de Toulouse (IRIT) de I'Université de Toulouse III et soutenue par le CNRS (http://osirim.irit.fr/site/). 


\section{Julien FIGEAC, Pierre RATINAUD, Nikos SMYRNAIOS, Guillaume CABANAC, Ophélie FRAISIER-VANNIER, Tristan SALORD et Fanny SEFFUSATTI}

du PS en repérant le groupe de comptes Twitter qui contient les tweets émis par le candidat Benoît Hamon et les autres cadres du Parti socialiste. Au terme de ce travail, une vérification manuelle a été réalisée pour tester la robustesse de cette méthode de détection des communautés et pour décrire plus précisément la composition de ce jeu de données (Fraisier et al., 2018).

\subsection{Méthode de catégorisation des types de contenus et de leur fiabilité}

Après avoir identifié les cinq principales communautés politiques qui ont investi Twitter durant la campagne présidentielle française de 2017, nous avons dû identifier les types d'information qu'elles ont partagés pour analyser la circulation des sources d'informations peu fiables. II apparaît alors que seulement un tiers des tweets et des retweets $(30,82 \%)$ contient une URL, un lien qui pointe vers une source d'information extérieure à Twitter (figure 1).

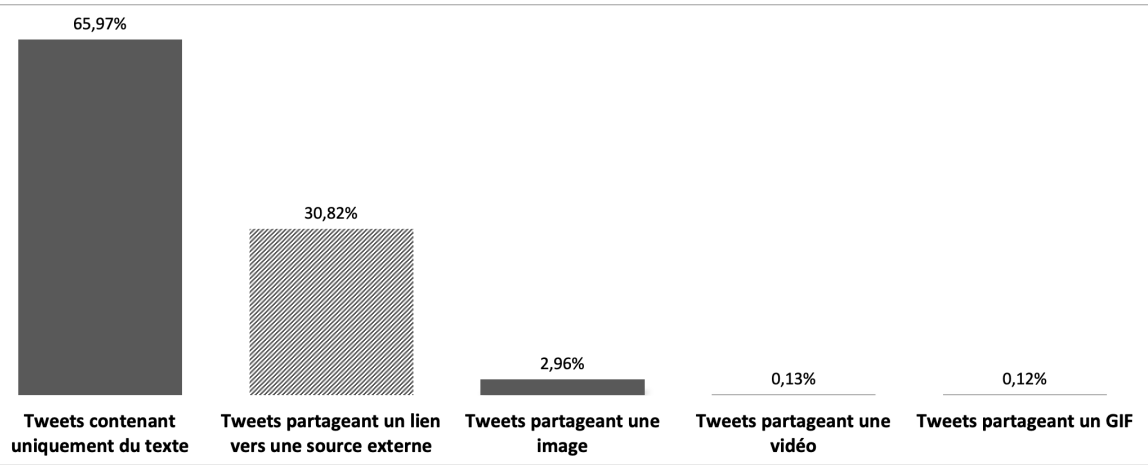

Figure 1. Part des différents types de contenus des tweets et des retweets

Dans le cadre de cet article, nous avons uniquement analysé ce sous-corpus de messages renvoyant à une source d'information extérieure $(n=13044619)$. Comme ces tweets contiennent des millions de liens, le premier objectif de cette recherche a consisté à identifier les principaux types de sources d'information qui ont été partagés : des articles de presse, des sondages, des vidéos YouTube, des billets des partisans, etc. 
Les téléphones mobiles, un outil de désinformation ? La circulation des informations peu fiables dans Twitter lors de la campagne présidentielle française de 2017

Pour atteindre cet objectif, nous avons réalisé un codage manuel des données. Nous avons tout d'abord distingué les informations produites par des organes de presse: les presses nationale, régionale et internationale, les magazines, les sites Web des chaînes de télévision et de radio, puis les pure players (c'est-àdire les titres de presse qui sont exclusivement diffusés sur le Web). Nous avons également catégorisé les informations diffusées par les fournisseurs d'accès à Internet (FAl) et les instituts de sondages, ainsi que l'ensemble des contenus diffusés par les partis et les institutions politiques, les sites de militants, les blogues et les pages personnelles, ainsi que les contenus partagés depuis des librairies de contenus (tracts, programmes politiques, etc.). Pour finir, nous avons identifié les informations publiées dans Twitter depuis un autre média social (Facebook, YouTube, Instagram, etc.). Cette catégorisation manuelle de ces diverses sources d'information est ainsi suffisamment inclusive et fouillée pour nous permettre de rendre compte du pluralisme de l'information durant cette campagne présidentielle, afin notamment de situer la place des organes de presse et des sources d'information " alternatives » dans les pratiques d'infomédiation au sein de Twitter.

Après avoir identifié les différents types d'information, nous avons dû identifier le degré de fiabilité de ces différentes sources. Pour atteindre ce second objectif, les chercheurs utilisent habituellement les données des sites de fact-checking ${ }^{6}$ et les milliers de fausses informations qu'elles recensent (Vosoughi, Roy et Aral, 2018). II n'existe qu'un seul site qui documente les fausses informations rédigées en français et relatives au contexte politique français. II s'agit du Décodex, qui a été développé par les journalistes du quotidien Le Monde ${ }^{7}$ (Figueira et Oliveira, 2017). Cette base de données référence les principales sources d'information qui circulent au sein de l'espace public numérique francophone et les classe en fonction de leur fiabilité. Elle analyse, identifie et distingue les sources fiables, qui réalisent un réel travail journalistique de vérification, des sources peu ou pas fiables, qui ont déjà relayé de la mésinformation ou de la désinformation. Le Décodex propose donc une base de données téléchargeable qui référence les

6 Parmi ces sites Web, on trouve: snopes.com, politifact.com, factcheck.org, truthorfiction.com, hoax-slayer.com et urbanlegends.about.com.

${ }^{7}$ Cf. le site du Décodex : https://www.lemonde.fr/verification/. 


\section{Julien FIGEAC, Pierre RATINAUD, Nikos SMYRNAIOS, Guillaume CABANAC, Ophélie FRAISIER-VANNIER, Tristan SALORD et Fanny SEFFUSATTI}

noms de domaine des différentes sources d'information en les catégorisant comme «Fiable » ou " Peu Fiable ».

Grâce à cette base de données, nous avons pu répondre à nos questions de recherche en évaluant si les téléphones mobiles tendent à favoriser la circulation des informations peu fiables et peuvent, par là même, amplifier la tendance de certaines communautés politiques à partager un plus grand nombre de fausses informations. Nous avons fait le choix de baser cette recherche sur cette base de données pour des raisons de faisabilité : il ne nous était pas possible de réaliser par nous-mêmes ce référencement de la fiabilité des sources d'information en identifiant lesquelles ont pu publier, au fil des années, de fausses informations.

Nous avons dès lors utilisé la base de données du Décodex pour identifier "automatiquement " les types et la fiabilité de sources partagées dans les tweets de notre corpus. Lors de cette procédure de détection automatisée, il s'est avéré que le nombre de sources détectées pouvait être amélioré s'il était complété par un codage manuel visant à identifier "qualitativement " les multiples déclinaisons que peuvent prendre les URL d'un même nom de domaine.

Au cours de ce travail de codage, nous avons également identifié les types de sources d'information qui ne sont pas répertoriés par le Décodex : les sites des partis politiques, des ONG, des associations, les blogues des militants, les nouvelles diffusées par les fournisseurs d'accès à Internet (Orange, SFR, Free, etc.), les plateformes de partage de données (Dropbox, etc.). L'objectif de ce codage était de nous permettre d'analyser en détail le pluralisme des informations tweetées lors de cette campagne. En revanche, il ne nous a pas été possible de nous prononcer sur la fiabilité de ces informations qui n'étaient pas référencées dans le Décodex, car il est difficile de dire si les informations partagées par certaines pages des partis politiques, des groupes de militants ou des blogues sont fiables ou non. Dans le cadre de cet article, l'analyse de la fiabilité des sources d'information ne repose donc que sur le classement proposé par les journalistes du Décodex. 
Les téléphones mobiles, un outil de désinformation ? La circulation des informations peu fiables dans Twitter lors de la campagne présidentielle française de 2017

\subsection{Méthode d'identification de la plateforme}

Après avoir identifié les principales communautés politiques qui ont investi Twitter durant la campagne présidentielle française de 2017 et catégorisé les sources d'information qu'elles ont partagées, nous avons dû identifier les plateformes utilisées pour publier les tweets en distinguant les usages de l'application mobile de Twitter de ceux de la plateforme Internet.

Les données fournies par Twitter permettent d'identifier un très grand nombre de plateformes différentes («Twitter for iPhone ", "Twitter for Web client", etc.). Nous les avons classées en deux catégories afin de distinguer les usages de Twitter réalisés depuis les téléphones mobiles, nous les appelons «MOS » (Mobile Operating System), de ceux réalisés depuis un navigateur Web d'un ordinateur, que nous désignerons par " non MOS ». Même si les usages des tablettes tactiles, telles que les iPads, sont différents de ceux des téléphones mobiles, nous avons pris le parti de les associer à la catégorie des supports mobiles (MOS). Ce parti pris repose notamment sur le nombre limité de tweets publiés depuis les tablettes numériques $(2,75 \% ; 446299$ tweets) et le fait que les travaux antérieurs ne différencient pas ces deux types de terminaux mobiles (Nelson et Taneja, 2018).

Au terme de ces différentes étapes, nous avons réussi à coder 11048901 tweets parmi ceux de notre corpus qui contenaient un lien vers une source d'information (13044 619). Nous avons plus précisément réussi à identifier les différents types de sources d'information partagées depuis l'application mobile de Twitter (46,99 \% ; tableau 1) ou d'un navigateur Web (53,01\%). Ces données vont nous permettre de répondre à nos questions de recherche en analysant la diffusion des diverses sources d'information durant cette campagne présidentielle, afin notamment de voir comment les sources d'information alternatives ont pu venir compléter ou concurrencer la place des organes de presse. 


\section{Julien FIGEAC, Pierre RATINAUD, Nikos SMYRNAIOS, Guillaume CABANAC, Ophélie FRAISIER-VANNIER, Tristan SALORD et Fanny SEFFUSATTI}

Tableau 1. Score de la catégorisation des sources d'information selon les types de terminaux

\begin{tabular}{|c|c|c|c|}
\hline & MOS & Non MOS & Total \\
\hline Types de sources d'information & $46,99 \%$ & $53,01 \%$ & $100 \%$ \\
\hline$N$ & 5191868 & 5857033 & 11048901 \\
\hline $\begin{array}{l}\text { Fiabilité des sources } \\
\text { d'information selon les } \\
\text { communautés }\end{array}$ & $41,01 \%$ & $58,99 \%$ & $100 \%$ \\
\hline$N$ & 846009 & 1217057 & 2063066 \\
\hline
\end{tabular}

Nous avons ensuite évalué la fiabilité de ces sources en mobilisant la catégorisation du Décodex : 2063066 tweets ont été codés. Ce résultat est nettement inférieur au précédent. Comme nous l'avons précisé, il n'est pas toujours possible de préciser la fiabilité d'une source d'information, par exemple quand il s'agit du site d'un parti politique ou d'un blogue. Ce score est inférieur, car nous avons pris le parti, ici, de ne retenir que les tweets qui ont pu être affiliés à une communauté politique. Comme il est là aussi bien souvent difficile de coder l'affiliation politique d'un compte Twitter, le score de détection final est assez bas. Malgré cela, cet échantillon reste conséquent puisque nous allons pouvoir analyser la circulation des sources d'information peu fiables, et identifier le rôle des terminaux, au sein d'un corpus de plus de 2 millions de tweets partagés par les cinq principales communautés politiques françaises.

\section{Résultats}

\subsection{Les usages de l'application mobile de Twitter défavorisent les organes de presse}

Durant cette élection présidentielle, les internautes ont alimenté leurs échanges d'idées et de contenus autour d'une grande diversité de sources d'information (11048 901; cf. tableau 1). La figure 2 montre plus précisément que les articles produits par les organes de presse forment de très loin le principal type d'informations mobilisé par les participants à ces espaces numériques pour traiter des sujets politiques et discuter des programmes des candidats. 
Les téléphones mobiles, un outil de désinformation ? La circulation des informations peu fiables dans Twitter lors de la campagne présidentielle française de 2017

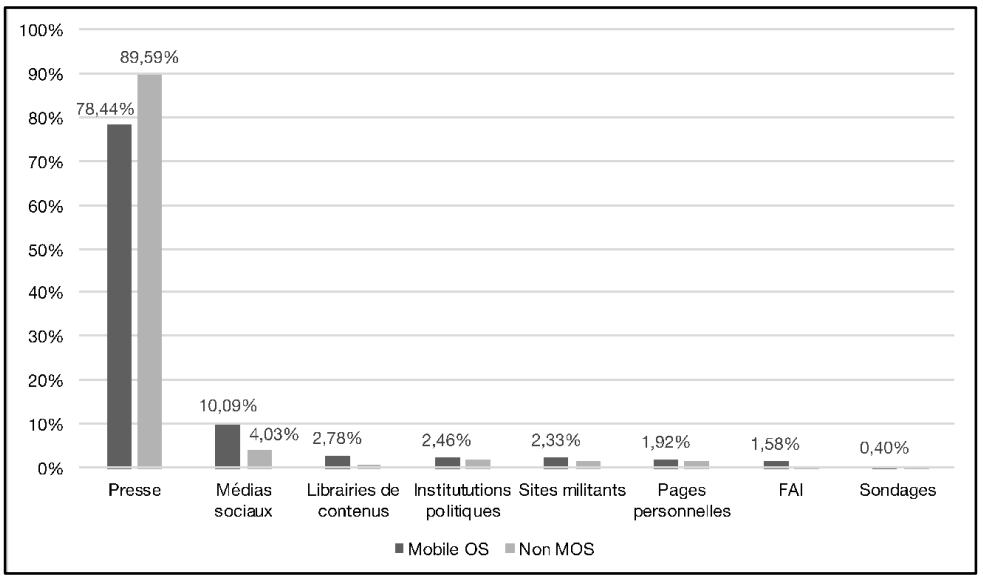

Figures 2. Types de sources partagées dans Twitter selon les plateformes

Cette tendance reste générale quelle que soit la plateforme utilisée - MOS ou Non MOS - pour partager des informations dans Twitter. Ceci étant dit, les données collectées mettent en évidence le fait que les usages de l'application mobile se traduisent par un taux de partage d'articles, publiés par la presse, inférieur, dans la mesure où la part de ces sources $(78,44 \%$; 1871 187) est moins importante de $11,15 \%$ (509 522) que la part de celles publiées depuis les plateformes Web $(89,59 \%$; $2380709)$.

La figure 3 ci-dessous aide à mieux comprendre ce phénomène en montrant que l'usage des différents types de plateformes n'influence en rien le type de presse partagé. La presse nationale reste la plus citée et représente environ la moitié des contenus de "Presse » qui ont circulé dans Twitter. Un quart de ces contenus provient ensuite des sites Web des chaînes de télévision (notamment les chaînes d'information en continu, les journaux télévisés des chaînes généralistes nationales et régionales) et, dans une moindre mesure, des stations de radio nationales. Le reste des contenus est issu des sites Web de magazines, de la presse internationale et régionale, ou des pure players. Précisons toutefois que les différentes communautés politiques ne mobilisent pas de la même manière ces différents types de presses. Les membres de la communauté FN ont, par exemple, tendance à davantage partager les nouvelles diffusées par les chaînes d'information en continu et les quotidiens régionaux. 


\section{Julien FIGEAC, Pierre RATINAUD, Nikos SMYRNAIOS, Guillaume CABANAC, Ophélie FRAISIER-VANNIER, Tristan SALORD et Fanny SEFFUSATTI}

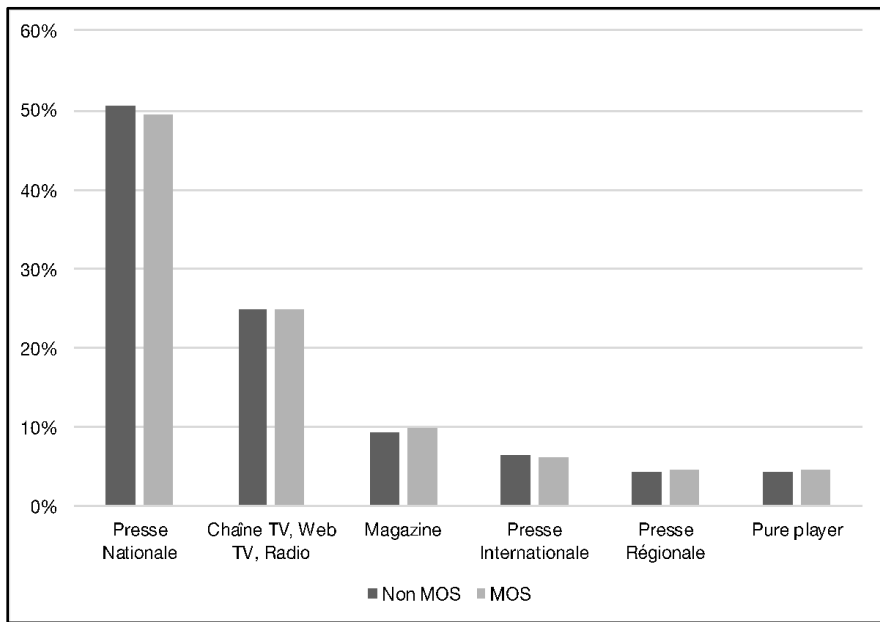

Figures 3. Types de presse partagés dans Twitter selon les plateformes

Pour comprendre pourquoi l'usage des téléphones mobiles défavorise sensiblement le recours aux contenus publiés par la presse journalistique, il faut revenir à la figure 2. Elle montre en effet une surreprésentation des contenus relayés dans Twitter depuis les applications mobiles des autres médias sociaux $(10,09 \%)$ : il s'agit essentiellement de vidéos YouTube $(69,25 \%)$ et de publications Facebook $(25,09 \%)$. Dans la mesure où les téléphones mobiles optimisent l'interopérabilité entre ces applications des médias sociaux, notamment par le design de leurs dispositifs de notification (Figeac et Chaulet, 2018), on peut considérer qu'ils favorisent davantage ces logiques d'usages entrelacées en comparaison avec les navigateurs Web. Le design de cette interopérabilité peut dès lors contribuer à expliquer pourquoi les utilisateurs de ces plateformes mobiles font davantage circuler des contenus entre les différents médias sociaux et pourquoi le design de ces outils tend à défavoriser la citation des titres de la presse journalistique.

La figure 2 montre ensuite que toutes les autres sources d'information sont légèrement surreprésentées dans les usages de cette plateforme, que ce soit les sites Web des institutions politiques ou des réseaux de militants, les blogues et les pages personnelles, les pages des instituts de sondage, etc. On peut entre autres relever la surreprésentation des libraires de contenus (Google photos, Dropbox, OneDrive, etc.) qui 
Les téléphones mobiles, un outil de désinformation ? La circulation des informations peu fiables dans Twitter lors de la campagne présidentielle française de 2017

représentent 2,78\% (219 443 tweets) des contenus partagés via les téléphones mobiles contre 0,82\% (101447 tweets) de ceux relayés depuis un navigateur Web. On peut considérer que leur usage, et notamment le recours à des services de stockage de photographies en ligne, est davantage pertinent depuis un téléphone mobile pour compenser, par exemple, leur plus faible capacité de stockage. Un autre résultat va en ce sens : le fait qu'il y a eu un peu plus de tweets (103 301) émis depuis une application mobile qui ont relayé un article publié par un FAl. Comme les quotidiens nationaux incitent souvent les utilisateurs à installer leurs applications sur leurs téléphones pour les consulter, beaucoup contournent cette contrainte d'utilisation en relayant plutôt des contenus de presse via les services des infomédiaires (Rebillard et Smyrnaios, 2010), tels que les Google News ou les nouvelles diffusées sur les sites des opérateurs téléphoniques. Précisons toutefois que ce résultat n'est pas précis : si les données Twitter précisent le nom du titre de presse partagé, elles ne permettent que très rarement d'identifier depuis quels infomédiaires les utilisateurs les ont initialement découverts.

L'ensemble de ces résultats corrobore les observations des experts du champ journalistique qui ont identifié, au moment des élections françaises, la place occupée dans les médias sociaux par les sources d'information alternatives et "non traditionnelles ${ }^{8}$ qui viennent concurrencer la centralité des informations produites par les organes de presse. On peut considérer que ces sources alternatives favorisent davantage la circulation de contenu peu fiable dans la mesure où elles ne sont pas forcément régulées par des professionnels de l'information, comme c'est par exemple le cas avec les pages des réseaux d'activistes ou les blogues de partisans.

Après avoir montré dans cette première partie une surreprésentation de ces sources alternatives dans les usages de l'application mobile de Twitter, nous allons maintenant chercher à approfondir ce résultat en nous demandant si cette surreprésentation va de pair avec une surreprésentation de la désinformation parmi les contenus partagés depuis les téléphones mobiles.

8 Cf. Haski, P. (2017). Patterns of disinformation in the 2017 French Presidential Election, Report. Repéré à https://www.bakamosocial.com/frenchelection/ 
Julien FIGEAC, Pierre RATINAUD, Nikos SMYRNAIOS, Guillaume CABANAC, Ophélie FRAISIER-VANNIER, Tristan SALORD et Fanny SEFFUSATTI

\subsection{L'application mobile de Twitter favorise la circulation des sources peu fiables dans certaines communautés politiques}

$\mathrm{Au}$ terme de notre travail, il apparaît une très nette surreprésentation des sources d'information fiables parmi celles tweetées durant la présidentielle de 2017 (figure 4). Les utilisateurs de ce média social l'exploitent donc très majoritairement pour partager des informations vérifiées qui ont été publiées par des professionnels de l'information. Ce constat diffère cependant sensiblement d'une plateforme à l'autre puisque la part des sources peu fiables partagées depuis l'application mobile de Twitter est supérieure de 4,55\% (figure 4). Les tests statistiques renforcent ce constat là où les résidus standardisés démontrent l'attraction entre ces deux modalités, entre le plus grand nombre de sources d'information peu fiables et l'usage des terminaux mobiles (tableau 2).

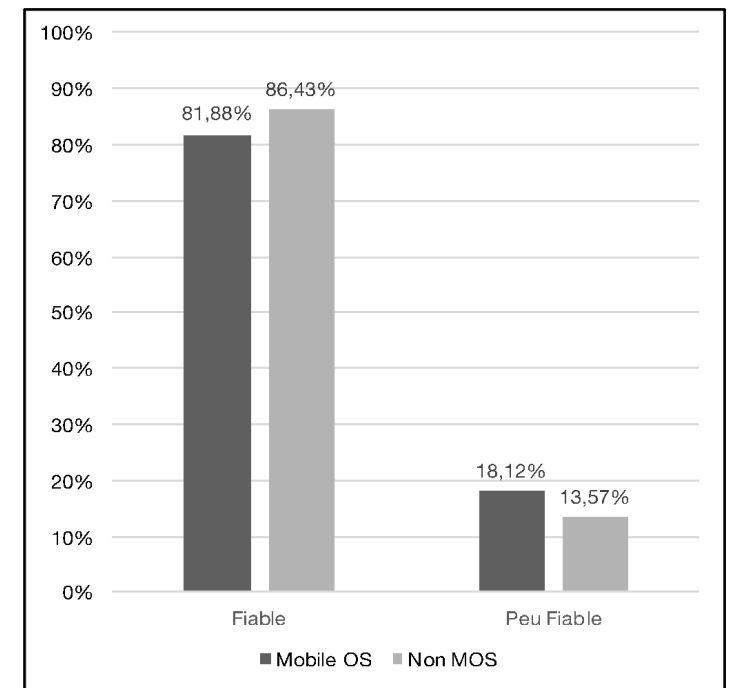

Figure 4. Fiabilité des sources partagées dans Twitter selon les plateformes 
Les téléphones mobiles, un outil de désinformation ? La circulation des informations peu fiables dans Twitter lors de la campagne présidentielle française de 2017

Tableau 2. Résultats des tests statistiques de corrélation

\begin{tabular}{llll} 
& & \multicolumn{2}{l}{ Fiabilité } \\
\cline { 3 - 4 } & & Fiable & Peu fiable \\
\hline Platefomes & $7933,9^{* \star *}$ \\
Résidus & Mobile & \\
standardisés & OS & $-89,07429$ & 89,07429 \\
& Non MOS & 89,07429 & $-89,07429$ \\
\hline $\mathrm{N}$ & 1744591 & 318,475 \\
\hline Test du Khi-deux : $\left.{ }^{*} \mathrm{p}<.05 ;{ }^{* *} p<.01 ;{ }^{* * *} \mathrm{p}<.001\right)$ &
\end{tabular}

On peut expliquer et contrôler la robustesse de ce résultat en évaluant si ces observations varient entre les différentes communautés politiques. La figure 5 ci-dessous montre ainsi qu'il existe de réelles disparités dans la mesure où le Front national partage un nombre significativement plus élevé de sources d'information peu fiables. Les pratiques d'échange d'information de la communauté des sympathisants du parti Les Républicains va également en ce sens, mais dans une bien moindre mesure. Ces observations rejoignent les travaux antérieurs qui ont identifié cette tendance auprès de ces deux communautés politiques (Gaumont, Panahi et Chavalarias, 2018).

Nous complétons ici ces travaux en montrant que la part des sources peu fiables partagées par les sympathisants FN et LR est bien supérieure à celle des autres communautés (cf. figures $5 a$ et $5 b$ ), mais cette tendance est d'autant nette dans notre étude avec le FN, car les résidus standardisés démontrent l'attraction entre ces deux modalités, entre cette communauté politique et le plus grand nombre de sources d'information peu fiables (cf. tableau 3).

Nos résultats montrent ensuite que l'usage de l'application mobile de Twitter renforce sensiblement la propension de ces deux communautés politiques à partager un nombre plus élevé de sources d'information peu fiables. Ceci est d'autant plus visible au FN puisque les sources peu fiables relayées depuis l'application mobile de Twitter $(47,48 \%$; cf. figure $5 \mathrm{~b})$ sont supérieures d'environ $10 \%$ à celles relayées depuis un navigateur Web $(37,52 \%$; figure $5 a)$. 


\section{Julien FIGEAC, Pierre RATINAUD, Nikos SMYRNAIOS, Guillaume CABANAC, Ophélie FRAISIER-VANNIER, Tristan SALORD et Fanny SEFFUSATTI}

II convient cependant de relativiser ce constat, car cette figure montre que le type de plateformes, mobiles ou non, n'a quasiment pas d'effet sur la part des informations, fiables ou non, partagées par les autres communautés politiques (LFI, PS, REM). Ce résultat nous invite dès lors à considérer que l'usage de la téléphonie mobile favorise la circulation des sources les moins fiables au sein des communautés politiques qui tendent dans l'absolu à en partager un nombre plus important, par ailleurs, depuis les autres terminaux.

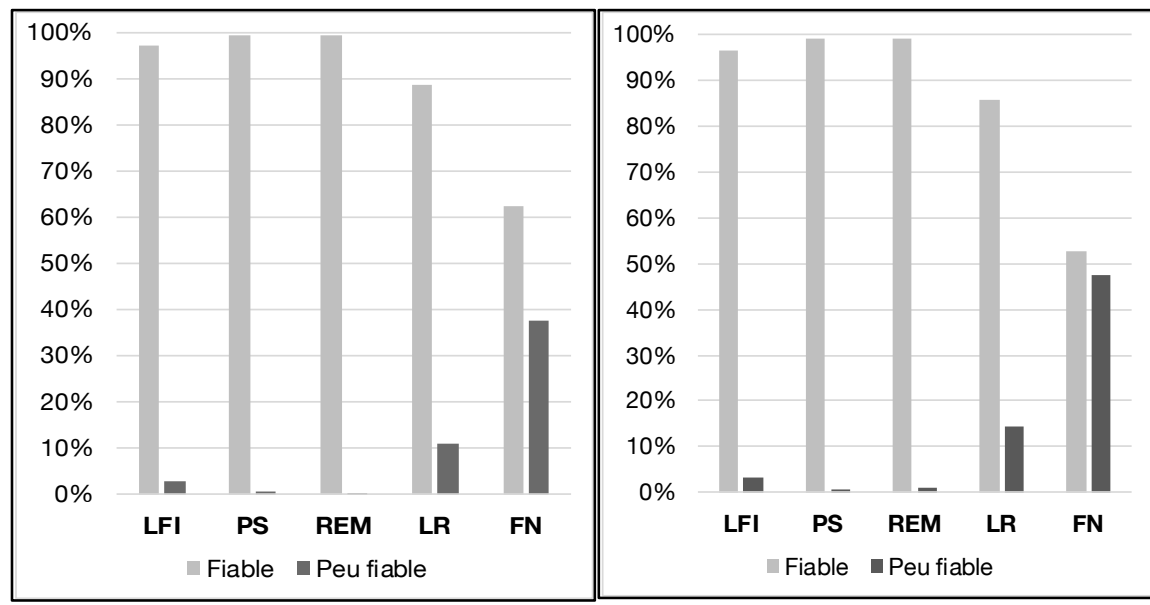

Figure 5. Fiabilité des sources partagées par les communautés politiques depuis les plateformes « Mobiles OS » et « Non Mobile»

Figure 5a. « Mobiles OS »

Figure 5b. « Non Mobiles » 
Les téléphones mobiles, un outil de désinformation ? La circulation des informations peu fiables dans Twitter lors de la campagne présidentielle française de 2017

Tableau 3. Résultats des tests statistiques de corrélation

\begin{tabular}{|c|c|c|c|c|c|}
\hline & \multicolumn{2}{|l|}{ Mobile OS } & \multicolumn{2}{|c|}{ Non Mobile OS } \\
\hline & & Fiable & Peu fiable & Fiable & Peu fiable \\
\hline \multicolumn{2}{|c|}{$\begin{array}{l}\text { Communauté } \\
\text { politique }\end{array}$} & \multicolumn{2}{|l|}{$198870^{* * *}$} & \multicolumn{2}{|l|}{$200710^{* \star \star}$} \\
\hline \multicolumn{2}{|c|}{ V de Cramer } & \multicolumn{2}{|c|}{$0,4848325^{\star *}$} & \multicolumn{2}{|c|}{$0,4060956^{* *}$} \\
\hline \multirow{5}{*}{ 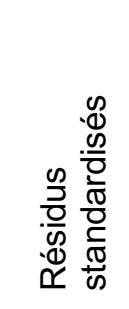 } & LFI & 198,8682 & $-198,8682$ & 149,3756 & $-149,3756$ \\
\hline & PS & 126,5958 & $-126,5958$ & 123,9352 & $-123,9352$ \\
\hline & REM & 141,2599 & $-141,2599$ & 176,9285 & $-176,9285$ \\
\hline & LR & 59,67161 & $-59,67161$ & 60,30241 & $-60,30241$ \\
\hline & FN & $-428,8489$ & 428,8489 & $-425,5135$ & 425,5135 \\
\hline \multicolumn{2}{|l|}{$N$} & 692,675 & 153,334 & 1051916 & 165,141 \\
\hline
\end{tabular}

Test du Khi-deux : $\left.{ }^{*} p<.05 ;{ }^{* *} p<.01 ;{ }^{* * *} p<.001\right)$

Test du V de Cramer : ${ }^{*} p<.1 ;{ }^{* *} p<.4 ;{ }^{* \star *} p<.8$

Pour affiner l'explication de ces résultats, il est pertinent d'évaluer si cette surreprésentation des sources peu fiables, qui ont été partagées via l'application mobile de Twitter, va de pair avec une surreprésentation des sources d'information " alternatives ». Les figures 6 présentent ce résultat en distinguant la presse journalistique des autres sources d'information ${ }^{9}$. Ces résultats montrent que le Front national mobilise davantage les sources d'information alternatives, que ce soit des sites Web de réseaux d'activistes ou des pages personnelles de militant, notamment depuis l'application mobile de Twitter $(42,78 \%$; figure $6 a)$. L'analyse des résidus standardisés confirme ce résultat (tableaux $4 a$ et $4 b$ ).

\footnotetext{
${ }^{9}$ À la différence de la figure 4, analysée dans la première partie, toutes les sources alternatives ont été agrégées ici. De plus, pour ne pas biaiser le résultat et comme nous l'avons précisé dans le volet méthodologique, les médias sociaux ont également été isolés dans la mesure où il ne nous est pas possible de les considérer comme des médias étant en eux-mêmes fiables ou non.
} 
Julien FIGEAC, Pierre RATINAUD, Nikos SMYRNAIOS, Guillaume CABANAC, Ophélie FRAISIER-VANNIER, Tristan SALORD et Fanny SEFFUSATTI

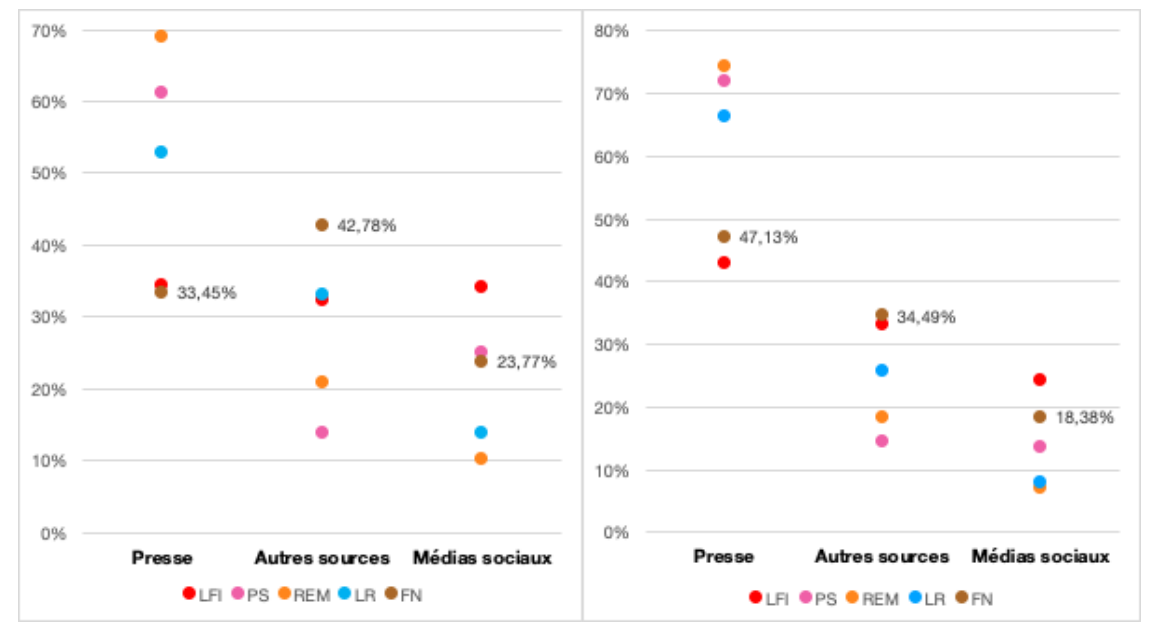

Figure 6. Types de contenus partagés par les communautés politiques selon les types de plateformes

Figure 6a. « Mobiles OS »

Figure 6b. « Non Mobiles »

Tableau 4a. Résultats des tests statistiques de corrélation (Mobile OS)

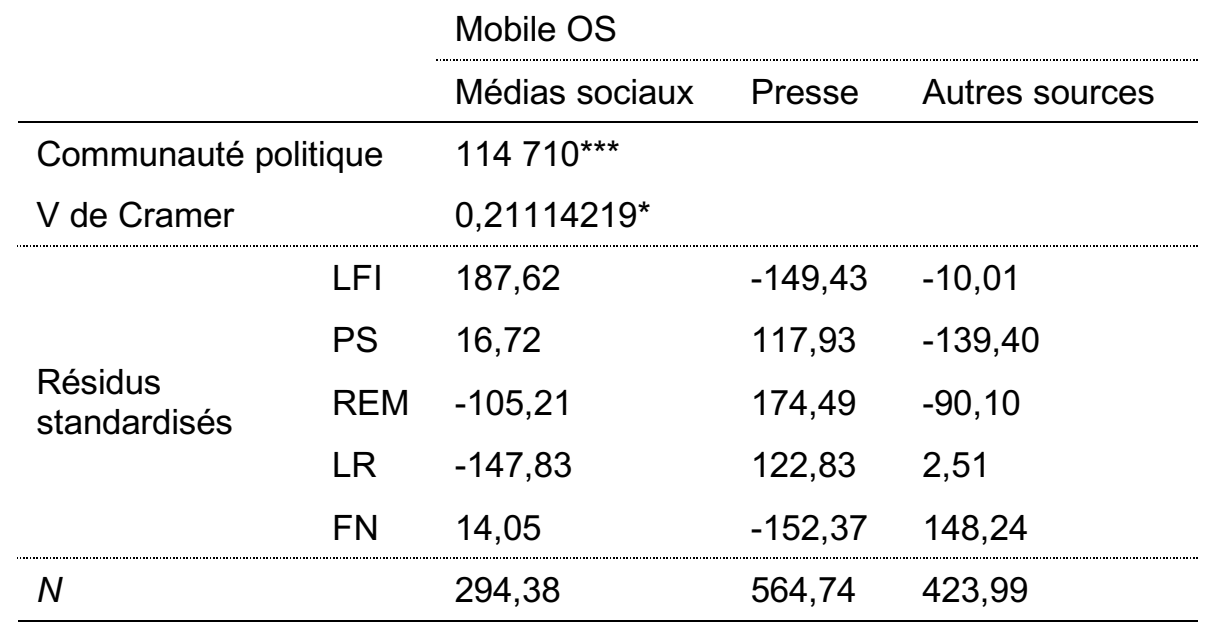

Test du Khi-deux : $\left.{ }^{*} p<.05 ;{ }^{* *} p<.01 ;{ }^{* * *} p<.001\right)$

Test du V de Cramer : ${ }^{*} p<.1 ;{ }^{* *} p<.4 ;{ }^{* \star *} p<.8$ 
Les téléphones mobiles, un outil de désinformation ? La circulation des informations peu fiables dans Twitter lors de la campagne présidentielle française de 2017

Tableau 4b. Résultats des tests statistiques de corrélation (Non Mobile OS)

\begin{tabular}{lllll}
\multicolumn{5}{c}{ Non Mobile OS } \\
\cline { 2 - 4 } & Médias sociaux & Presse & Autres sources \\
\hline Communauté politique & $109920^{* * *}$ & & \\
V de Cramer & $0,1886985^{*}$ & & \\
\hline & LFI & 174,20 & $-189,58$ & 74,51 \\
& PS & $-2,01$ & 95,67 & $-104,04$ \\
Résidus & REM & $-92,98$ & 153,50 & $-97,51$ \\
standardisés & LR & $-157,67$ & 142,07 & $-34,84$ \\
& FN & 94,86 & $-169,72$ & 113,97 \\
& & 212,98 & 907,93 & 422,51 \\
\hline$N$ & & &
\end{tabular}

Test du Khi-deux : $\left.{ }^{*} p<.05 ;{ }^{* *} p<.01 ;{ }^{* * *} p<.001\right)$

Test du V de Cramer : ${ }^{*} p<.1 ;{ }^{* *} p<.4 ;{ }^{* \star *} p<.8$

Dans le prolongement de la première partie, ces résultats montrent donc que la proportion des sources peu fiables en circulation dans une communauté politique tend à être corrélée à la proportion de ces sources alternatives, produites par des individus extérieurs au champ journalistique. Comme les utilisateurs de l'application mobile de Twitter relaient plus couramment ces sources alternatives, cette recherche montre que la participation aux débats et aux échanges d'information à partir de l'application mobile de Twitter peut favoriser la citation et la circulation de sources d'information peu fiables et, de fait, la propagation d'informations fausses ou erronées. Cependant, cette étude montre que ce phénomène concerne essentiellement deux communautés politiques françaises, celle de la droite (LR) et de l'extrême droite (FN).

\section{Conclusion}

De nombreuses recherches fournissent des éléments d'explication pertinents pour comprendre la manière dont les médias sociaux peuvent favoriser la propagation de la désinformation et ainsi influer sur les équilibres politiques des démocraties libérales. Face aux enjeux soulevés par ce phénomène, la singularité des recherches françaises tient en grande partie à la place centrale, pour ne pas dire croissante, 


\section{Julien FIGEAC, Pierre RATINAUD, Nikos SMYRNAIOS, Guillaume CABANAC, Ophélie FRAISIER-VANNIER, Tristan SALORD et Fanny SEFFUSATTI}

qu'occupent dorénavant l'extrême droite et les communautés politiques radicalisées dans le champ politique français. Cette singularité du contexte français, notamment le fait que la candidate de l'extrême droite a accédé au second tour de l'élection présidentielle de 2017, conduit les travaux français à compléter les recherches scientifiques internationales sur un versant de ce phénomène qu'elles explorent moins directement, du fait souvent de la structuration bipartite de leurs sociétés, à savoir une meilleure compréhension du fonctionnement des groupes politiques extrémistes dans la polarisation idéologique des opinions au sein des médias sociaux (Bond et Messing, 2015). Ce contexte politique est central pour comprendre pourquoi l'extrême droite est au cœur des autres recherches françaises abordant ces questions (Gaumont, Panahi et Chavalarias, 2018). II en va de même ici dans la mesure où cet article révèle que les usages de l'application mobile de Twitter favorisent avant tout la circulation des sources peu fiables au sein de la communauté politique de l'extrême droite française. Cette question du rôle des téléphones mobiles dans la propagation de la désinformation mériterait donc d'être posée dans d'autres contextes politiques, notamment ceux qui sont structurés autour d'un bipartisme.

Les recherches anglophones ont également la possibilité de mobiliser les ressources de différents sites de fact-checking qui recensent des milliers de fausses informations rédigées en langue anglaise (Vosoughi, Roy et Aral, 2018). II n'existe en France qu'une seule base de données disponible, le Décodex. Elle soulève un certain nombre de problèmes découlant tout d'abord du fait qu'elle soit alimentée par des employés du journal Le Monde. Ils sont effectivement juges et partis lorsqu'ils évaluent et déterminent la fiabilité des informations publiées par les autres médias et une diversité toujours plus grande de sites Web. II en va de même lorsqu'ils se positionnent par rapport à l'affiliation politique de ces sources d'information en précisant si elles sont " plutôt de droite " ou "plutôt de gauche ". Si cette limite mérite d'être rappelée, il convient également de préciser à quel point il est impossible, pour un collectif de chercheurs, de pouvoir alimenter une base de données référençant, jour après jour, les sources d'information " peu fiables » qui ont pu publier ou relayer des fausses informations. Le recours à ce type de 
Les téléphones mobiles, un outil de désinformation ? La circulation des informations peu fiables dans Twitter lors de la campagne présidentielle française de 2017

bases de données de seconde main, alimentées par des professionnels du fact-checking, constitue la norme; il forme probablement même aujourd'hui une condition incontournable pour les chercheurs qui veulent analyser le phénomène de la désinformation au sein du Web francophone. Le Décodex pose également problème $d u$ fait même d'être alimenté « manuellement » par des journalistes sans l'appui d'algorithmes ou de formes automatisées de traitement de l'information (Figueira et Oliveira, 2017). C'est pourquoi nous avons pris le parti de compléter ces données par un codage manuel, notamment pour affiner la catégorisation des sources d'information alternatives animées par des militants ou des groupes d'activistes. Cette démarche est importante, car ces sources jouent un rôle central dans la propagation de la désinformation.

Elles renvoient à des contenus produits par des acteurs qui cherchent à promouvoir des formes de journalisme citoyen. Ce journalisme favorise le pluralisme de l'information dans les démocraties, sans pour autant renvoyer à des initiatives purement "alternatives » en raison du rôle de certains journalistes professionnels (Goode, 2009). Ce journalisme citoyen est d'autant plus important quand on s'intéresse au rôle de la téléphonie mobile tant ces supports favorisent ces modes d'expression alternatifs, notamment lors des rassemblements et des manifestations (Tufekci, 2019). II existe dès lors un réel enjeu concernant la classification de la fiabilité des informations que les militants politiques ou des " médiactivistes » (Cardon et Granjon, 2013) peuvent partager via l'application mobile de Twitter ou leurs blogues. II est problématique de catégoriser toutes ces informations alternatives comme étant peu fiables, en partant du principe qu'elles n'ont pas été produites par des journalistes ou qu'elles adoptent un positionnement trop critique, trop contre-hégémonique. L'enjeu, aujourd'hui, consiste donc à mieux évaluer la fiabilité de ces sources alternatives pour mieux détecter les modes de propagation de la désinformation. Cet enjeu est d'autant plus important quand il s'agit de comprendre la manière dont les téléphones mobiles peuvent favoriser ce phénomène, car, comme cette recherche le montre, ils détournent de l'usage captif des applications de la presse quotidienne en favorisant plutôt le partage d'informations produites par ce type de sources alternatives. 


\section{Julien FIGEAC, Pierre RATINAUD, Nikos SMYRNAIOS, Guillaume CABANAC, Ophélie FRAISIER-VANNIER, Tristan SALORD et Fanny SEFFUSATTI}

La démocratisation des téléphones mobiles soulève ainsi ce double enjeu, auquel les recherches futures devront répondre, relatif au rôle de ces outils dans la production d'informations alternatives et citoyennes, capturées sur le vif des événements, puis dans leur propagation à grande échelle sur les médias sociaux (Tufekci, 2019). Si les ressources expressives de la téléphonie mobile favorisent la participation citoyenne et le pluralisme de l'information, à partir de quels moments offrentelles à des militants radicalisés une liberté d'expression contraire aux règles du jeu démocratique?

\section{Références}

Aker, J. C., Collier, P. et Vicente, P. C. (2017). Is information power? Using mobile phones and free newspapers during an election in Mozambique. Review of Economics and Statistics, 99(2), 185-200.

Allcott, H. et Gentzkow, M. (2017). Social media and fake news in the 2016 election. Journal of economic perspectives, 31(2), 211-236.

Bond, R. et Messing S. (2015). Quantifying social media's political space: Estimating ideology from publicly revealed preferences on Facebook. American Political Science Review, 109(1), 62-78.

Cardon, D et Granjon, F. (2013). Médiactivistes. Paris, France : Presses de Sciences Po.

De Domenico, M., Lima, A., Mougel, P. et Musolesi, M. (2013). The anatomy of a scientific rumor. Scientific reports, (3). https://doi.org/10.1038/srep02980

Figeac, J. (2007). La configuration des pratiques d'information selon la logique des situations. Réseaux, 25(143), 17-44.

Figeac, J. et Chaulet, J. (2018). Video-ethnography of social media apps' connection cues in public settings. Mobile Media et Communication, 6(3), 407-427.

Figueira, Á. et Oliveira, L. (2017). The current state of fake news: Challenges and opportunities, Procedia Computer Science, (121), 817-825. 
Les téléphones mobiles, un outil de désinformation ? La circulation des informations peu fiables dans Twitter lors de la campagne présidentielle française de 2017

Fraisier, O., Cabanac, G., Pitarch, Y., Besançon, R et, Boughanem, M. (2018, juin). \#Élysée2017fr: The 2017 French Presidential Campaign on Twitter. Communication présentée à la conférence International Conference on Weblogs and Social Media (ICWSM 2018), AAAI Press. Repéré à https://aaai.org/ocs/index.php/ICWSM/ICWSM18/ paper/view/17821

Gaumont, N., Panahi, M. et Chavalarias, D. (2018). Reconstruction of the socio-semantic dynamics of political activist Twitter networks: Method and application to the 2017 French presidential election. PLOS ONE, 13(9). https://doi.org/10.1371/journal.pone.0201879

Goode, L. (2009). Social news, citizen journalism and democracy. New media et society, 11(8), 1287-1305.

Grinberg, N., Joseph, K., Friedland, L., Swire-Thompson, B. et Lazer, D. (2019). Fake news on Twitter during the 2016 US presidential election. Science, 363(6425), 374-378.

Guess, A., Nagler, J. et Tucker, J. (2019). Less than you think: Prevalence and predictors of fake news dissemination on Facebook. Science advances, 5(1). doi: 10.1126/sciadv.aau 4586

Nelson, J. L. et Taneja, H. (2018). The small, disloyal fake news audience: The role of audience availability in fake news consumption. New Media \& Society, 20(10), 3720-3737.

Oh, O., Kwon, K. H. et Rao, H. R. (2010). An Exploration of Social Media in Extreme Events: Rumor Theory and Twitter during the Haiti Earthquake 2010. Icis, (231), 7332-7336.

Rebillard, F. et Smyrnaios, N. (2010). Les infomédiaires, au cœur de la filière de l'information en ligne. Réseaux, (160-161), 163194.

Smith, A. et Page, D. (2015). U.S. smartphone use in 2015. Repéré à http://www.pewlnternet.org/2015/04/01/ussmartphone-use-in-2015/

Smyrnaios, N. et Ratinaud, P. (2014), Comment articuler analyse des réseaux et des discours sur Twitter. tic\&société, 7(2), 120147. 
Julien FIGEAC, Pierre RATINAUD, Nikos SMYRNAIOS, Guillaume CABANAC, Ophélie FRAISIER-VANNIER, Tristan SALORD et Fanny SEFFUSATTI

Tandoc Jr, E. C., Lim, Z. W. et Ling, R. (2018). Defining "fake news" A typology of scholarly definitions. Digital journalism, 6(2), 137-153.

Tufekci, Z. (2019). Twitter et les gaz lacrymogènes. Forces et fragilités de la contestation connectée. Caen, France : C\&F Éditions.

Uberti, D. (2016) The Real History of Fake News. Columbia Journalism Review, (15). Repéré à https://www.cjr.org/special report/fake news history.php

Venkatraman, A., Mukhjia, D., Kumar, N. et Singh Nagpal, S. J. (2016) Zika virus mis-information on the Internet, Travel Medicine and Infectious Disease, 14(4), 421-2. doi: 10.1016/j.tmaid.2016.05.018

Venturini, T., Jacomy, M., Bounegru, L. et Gray, J. (2018). Visual Network Exploration for Data Journalists. Dans S. Eldridge II et B. Franklin (dir.), The Routledge Handbook to Developments in Digital Journalism Studies. Abingdon, Royaume-Uni : Routledge.

Vishwanath, A. (2016). Mobile device affordance: Explicating how smartphones influence the outcome of phishing attacks. Computers in Human Behavior, (63), 198-207.

Vosoughi, S., Roy, D. et Aral, S. (2018). The spread of true and false news online. Science, 359(6380), 1146-1151. doi: $10.1126 /$ science.aap9559

Wardle, C. et Derakhshan, H. (2017). Information Disorder: Toward an interdisciplinary framework for research and policymaking. Strasbourg, France : Council of Europe report.

Weedon, J., Nuland, W. et Stamos, A. (2017). Information operations and Facebook. S.I.: Facebook. Repéré à https://fbnewsroomus.files.wordpress.com/2017/04/facebook -and-information-operations-v1.pdf

Westlund, O. (2015). News consumption in an age of mobile media: Patterns, people, place, and participation. Mobile Media et Communication, 3(2), 151-159. 